\title{
TIEMPO, ESPACIO Y CIRCULACIÓN EN LA ERA DE LA GLOBALIZACIÓN
}

\author{
Susan PETRILLI \\ Universidad de Bari (Italia) \\ s.petrilli@lingue.uniba.it
}

Resumen: Se propone en este trabajo una reflexión semiótica en las condiciones de la globalización, describir su lógica y proponer una crítica. A la luz de esto, se examina la comunicación, que hoy es comunicación total, convergiendo con la estructura social y el mercado total. La lógica de la identitad se opone a la apertura hacia la alteridad.

Abstract: We propose a semiotic reflection on the conditions of globalization, describe its logic, and propose a critique. In this light we analyze communication, which today is total communication converging with social structure and total market. The logic of identity is juxtapposed to opening towards alterity.

Palabras clave: Globalización. Comunicación. Alteridad. Crítica semiótica. Sentir.

Key words: Globalization. Communication. Otherness. Critical Semiotics. Sensibility. 
Este trabajo retoma, para desarrollarlas, algunas secciones de un libro que escribí con Augusto Ponzio: Il sentire della comunicazione globale (en español podría traducirse como El concepto de comunicación global, o bien El sentir de la comunicación global). Fue publicado por la editorial Meltemi, en Roma, en el año 2000. En él, la investigación semiótica se orienta en esa dirección que la escuela de Bari ha llamado Semio-ética, un concepto, una palabra, que es también el título de otro libro mío y de A. Ponzio, un libro más reciente, de 2003, publicado igualmente en Roma, por Meltemi.

El trabajo que aquí presento se estructura en los siguientes temas: 1. Estética e ideo-lógica de la globalización; 2. El tiempo; 3. El tiempo del no-trabajo; 4. Espacio y circulación; 5 . El espacio urbano; 6 . El espacio entre indiferencia y diferencia museificada; 7 . Viabilidad del espacio y disponibilidad de medios de circulación personales; 8. Migración y alteridad irreducible a la comunidad del trabajo; 9. Sentirse extracomunitario; 10. Alteridad y proximidad entre migración y paro; 11 . La posibilidad de sentirse en el mundo, pero no del mundo de la globalización.

En la era de la globalización, es decir, en la era de la comunicación-producción hecha mundial, «mundializada», tanto la interculturalidad como la insularidad pueden orientarse, en mi opinión, sea en un sentido de cierre de la identidad, sea en un sentido de apertura hacia la alteridad.

Si tuviese que elegir una cita como epígrafe, tomaría, parafraseándola, una de Por quien doblan las campanas, de E. Hemingway, en la que se refieren unas palabras de John Donne, que dicen así:

Cada identidad individual o colectiva, aunque pueda ser concebida como una isla, formará parte siempre de un archipiélago. Y cuando alguna de estas identidades, individuales o colectivas, sufra o muera, por más lejana y ajena que a ti sea, estará sufriendo y muriendo algo tuyo. Por ello, no preguntes nunca por quién doblan a muerte las campanas, porque, de algún modo, tañen siempre también por $t i$.

\section{ESTÉTICA E IDEO-LÓGICA DE LA GLOBALIZACIÓN}

En la primera parte de la Crítica de la razón pura, en la «estética trascendental», con la que comienza la «Doctrina trascendental de los elementos», Kant escribe: 
Llamo estética trascendental a una ciencia de todos los principios a priori de la sensibilidad. Debe existir esa ciencia, una ciencia que constituya la primera parte de una doctrina trascendental de los elementos, en oposición a la que contiene los principios del pensamiento puro, y se denomina lógica trascendental (Kant, 1998: 66-67, de la trad. ${ }^{\circ}$ italiana).

Análogamente, podríamos decir que una reflexión semiótica sobre las condiciones fundamentales de la globalización que sea capaz de considerar no sólo aspectos parciales y sectoriales de la comunicación global, y que sea capaz de no quedarse empíricamente atada a sujetos psicológicos, sino que sea capaz, al contrario, de captar su lógica, y, por lo tanto, capaz de desarrollar su crítica, tiene que empezar por una estética trascendental, por una reflexión sobre el sentir no reflexivo, no-consciente, de la comunicación global, investigando los principios a priori de la sensibilidad de la globalización.

En nuestra estética trascendental de la globalización partiremos, como Kant, de las dos formas fundamentales de la intuición sensible: el espacio y el tiempo.

En nuestro caso, sin embargo, un caso en el que la estética concierne al sistema de la comunicación-producción global, resulta que, a diferencia de la crítica kantiana, no es posible separar los elementos de la sensibilidad de los principios que constituyen la lógica de tal sistema. Es decir, no es posible comprender la sensibilidad espacio-temporal más que desde el interior de la ideología de dicho sistema, y, por consiguiente, remontándose a su lógica. Podríamos incluso decir que se trata, en este caso, de reconocer la inseparabilidad de estética y lógica.

\section{EL TIEMPO}

A diferencia de la crítica kantiana, no comenzaremos con el concepto de espacio, sino con el de tiempo. En efecto, dado el carácter fundamental del tiempo en la lógica del sistema capitalista, la sensibilidad del espacio depende de la sensibilidad del tiempo. En la globalización, el tiempo es el tiempo de la comunicación-producción que caracteriza la actual fase de la forma de producción capitalista.

La comunicación de la forma social capitalista se caracteriza por la tendencia a realizarse de manera total, sea en cuanto afecta a la extensión, sea en cuanto afecta a lo que puede ser comunicado en el ámbito de la circulación de personas, bienes (y servicios) y mensajes. En la forma de producción ca- 
pitalista, como el intercambio es esencialmente intercambio de mercancías, la comunicación y el mercado coinciden; y la tendencia a la comunicación total es la tendencia al mercado total, sea como extensión, sea como posibilidad de transformación en mercancía cualquier cosa. Por consiguiente, también los mismos mensajes se vuelven mercancías, tal y como las mercancías no podrían ser mercancías si no fueran también mensajes. Como circulación de mensajes-mercancías además de mercancías-mensajes, y como circulación de personas por medios de circulación que también son mercancías, toda la comunicación resulta convertible en mercado.

Pero de los tres momentos del ciclo productivo — es decir, producción, intercambio (o circulación o mercado) y consumo-, la comunicación no afecta tan sólo al momento central del cambio: la misma producción tiende a realizarse como comunicación («automazione», empleo a distancia de la fuerza de trabajo, tele-trabajo); y hasta el mismo consumo, en cuanto consumo de mercancías-mensajes y mensajes-mercancías, es también proceso comunicativo.

La comunicación se presenta, pues, como la estructura social, y cada comportamiento individual o colectivo forma parte de programas, programaciones o maquinaciones de la comunicación de la forma capitalista de producción, y obedece a su (ideo)lógica.

Dándose la identificación de producción y comunicación, el ciclo entero productivo-comunicativo, producción-circulación-consumo, teniendo como fin la producción, tiene como fin la comunicación: la producción por la producción, o sea, la comunicación por la comunicación. La producción por el provecho, por las ganancias, desde luego, pero las ganancias, el provecho, están en función de la producción, es decir, de la reproducción ampliada del ciclo productivo-comunicativo y de la total red comunicativa de que la que forma parte y de la que depende por completo. Así que la clase dominante de la actual forma social capitalista es la clase que controla su comunicación.

El tiempo de la comunicación-producción, en cuanto tiempo de la forma social capitalista, se presenta como tiempo que hay que reducir. Pero nunca tanto como en esta fase actual esa reducción ha sido tan requerida y tan sentida como tal; nunca el tiempo se ha presentado como hoy, como algo que hay que eliminar. La tendencia de restricción, de limitación del tiempo, característica del sistema de producción capitalista, en la actualidad se acentúa hasta el paroxismo, por la unión entre el tiempo de trabajo y las ganancias o provecho. 
Además, la reducción del tiempo concierne a las tres fases del ciclo productivo. Por la inseparabilidad de la producción, circulación y consumo, para impedir la superproducción, a la reducción del tiempo de producción tiene que añadirse también la reducción del tiempo de circulación y de consumo, con la consiguiente aceleración de la comunicación dentro de estos otros dos momentos del ciclo productivo en su totalidad.

Esta agilización o aceleración se consigue por el uso funcional de la investigación científica y de la invención técnica al servicio de las exigencias de la producción capitalista. El objetivo es la reducción del tiempo total de la comunicación, es decir, del de la producción, del de la circulación, del del consumo.

La extensión de la reducción del tiempo de trabajo a la entera comunicación, conlleva que, también en la vida cotidiana fuera del lugar de trabajo y fuera del tiempo de trabajo, esté presente la tendencia a la eliminación del tiempo, a que el tiempo sea concebido como algo que debe eliminarse. (Advirtamos de pasada, sin embargo, la orientación de la comunicación-producción a que no haya un «lugar de trabajo» específico, a que no existan fronteras precisas entre trabajo y no-trabajo.)

\section{EL TIEMPO DE NO-TRABAJO}

La conversión en plusvalía de la reducción del tiempo de trabajo es una conversión conseguida por el desarrollo tecnológico. Esta plusvalía, este «plustrabajo» respecto al «intercambio equitativo» entre trabajo y capital, es una ganancia, un provecho que decide la configuración concreta del no-trabajo: tiempo libre, tiempo de espera del puesto de trabajo, tiempo de formación, tiempo excedente (tiempo sobrante, sin carga de trabajo, por falta de carga de trabajo) y tiempo de pérdida del trabajo, tiempo de fondo de desempleo, de pre-jubilación, de jubilación, etc.

El tiempo de no-trabajo es un tiempo subalterno al tiempo de trabajo; también cuando uno está en el paro está preocupado por el trabajo (también el tiempo de paro es subalterno al tiempo de trabajo). Es subalterno, ya sea como pausa o como descanso del trabajo, para recuperar la energía necesaria para él (que delimita el tiempo: el horario laboral, el calendario laboral, sus fiestas, las vacaciones, etc.), ya sea como tiempo de paro, de espera de trabajo (durante su búsqueda), ya sea, finalmente, como conclusión de la vida activa y laboral (jubilación, superación de los «límites de edad» en el curso de la «formación permanente» en espera de trabajo). 
Como tiempo libre, el tiempo de no-trabajo, es un tiempo vacío, un «tiempo perdido», un tiempo que hay que «llenar», «transcurrir», «pasar», «matar», o que hay que convertir en ganancias, en provecho (la industria del tiempo libre); y, como tiempo de paro, es un tiempo con el que hay que terminar lo antes posible. El tiempo libre, aunque sea «tiempo perdido» con respecto al tiempo productivo, tiene que ser, también él, economizado, porque obedece a la lógica productiva de no perder tiempo.

La agilización o aceleración de la comunicación del tiempo libre se sitúa en la agilización o aceleración de la circulación y del consumo, y las posibilidades de disfrutar de él son obviamente relativas a la ocupación, al tiempo de trabajo, y a su correspondiente tiempo de no-trabajo, el tiempo de descanso, de paro, pero también al grado de conversión del tiempo de no-trabajo en «plustrabajo», es decir, en plusvalía y en ganancias, en provecho. La aceleración de la comunicación del tiempo libre es también, por lo tanto, relativa al coste del trabajo.

El tiempo de no-trabajo es «esencial» en la forma social de producción capitalista, y se manifiesta como una difusa liberación del trabajo (del trabajo libre, del trabajo como mercancía), ya sea como paro, fondo de desempleo, reducción del horario de trabajo, jubilación anticipada, y ello como consecuencia de la automatización del sector productivo y del terciario; ya sea como posibilidad de otium, de «trabajo inmaterial», que la exgencia de conocimiento hecha por el capital, debido a la investigación científica y tecnológica, ha desarrollado involuntariamente. El tiempo de no-trabajo se presenta, de este modo, también como tiempo de aprendizaje, de estudio, de especialización, a consecuencia de la necesidad de uso por el capital, en la fase de comunicación-producción, del trabajo llamado «inmaterial», del «trabajo intelectivo», al que exige ser constantemente conforme, adecuado y funcional para el desarrollo científico y tecnológico.

El incremento del tiempo de no-trabajo resulta esencial, estructural, en la forma social capitalista, también desde el punto de vista de la destrucción que el desarrollo capitalista realiza con respecto a las ocupaciones tradicionales del «subdesarrollo» o de los países «en desarrollo». En el «tiempo de no-trabajo de la mayoría» - a diferencia de sólo en el de la minoría, como sucedía en fases anteriores-, tiempo que hoy el desarrollo capitalista conlleva, debe ser anotada también la pérdida de las condiciones de trabajo pertenecientes a formas sociales no capitalistas provocada por el desarrollo capitalista hasta reducir incluso a estar por debajo de las condiciones de subsistencia a cada vez mayor parte de la población mundial. Ello causa el fenómeno actual de la 
migración, no asumible por el capital ni como ocupación, ni como reserva de parados, a diferencia de la emigración tradicional (con el consiguiente «cierre comunitario» de fronteras a los «extracomunitarios»).

\section{ESPACIO Y CIRCULACIÓN}

El espacio debe ser considerado inseparablemente del tiempo de la forma social de producción capitalista. En la globalización, tal tiempo es el tiempo de la comunicación-producción.

El espacio de la comunicación global se caracteriza por la «penetrabilidad», la «conectabilidad», la «transitabilidad», la «viabilidad», la «accesibilidad». Son las características del «espacio» según la «ideo-lógica de la comunicación mundializada», que, por su adherencia al mundo tal como es, corresponden ciertamente a la realidad, pero, por eso mismo, también poseen sus contradicciones. Por tanto, tales características se traducen en sus contrarias. De tal modo, el sentir la «penetrabilidad», la «conectabilidad», la «transitabilidad», la «viabilidad», la «accesibilidad» del espacio se asocia con el sentir —mucho más atormentado, doloroso, injusto, frustrado, injusto, precisamente por su inevitable convivencia con lo anterior - la «impenetrabilidad», la «inconectabilidad», la in»transitabilidad», la «inviabilidad», la «inaccesibilidad».

El ejemplo más macroscópico de tal contradicción real de la ideo-lógica contemporánea se encuentra en las migraciones. A la vez que la tendencia a que las fronteras se abran totalmente a la circulación de mercancías, incluidas en ellas las fuerzas de trabajo, en cambio dichas fronteras se cierran a la $m i$ gración, admitiendo, a lo sumo, sólo el mínimo margen asimilable de ésta, el desplazamiento de la fuerza de trabajo asumible en el mercado nacional e internacional. La migración obliga al cierre del circuito de la circulación, de la comunicación, al cierre del «libre mercado» del trabajo, a la imposibilidad de extensión ilimitada de la categoría de trabajo en general, y de la transformación de la persona en fuerza de trabajo. La homologación inherente al «intercambio equitativo», homologación que hace posible la abstracción, la equiparación, la commensurabilidad, el intercambio, la comunicación, se atasca ante la migración. El fenómeno actual de la migración es al mismo tiempo nuevo y antiguo, pero ciertamente no se deja identificar con la emigración, cuya característica principal es su carácter englobable en el sistema de producción, su «englobabilidad». La especificidad de la migración es, en cambio, el hecho que nos sitúa ante una alteridad no asimilable. 
A la economización del tiempo - característica de la forma capitalista exasperada de la fase actual de comunicación-producción-, gracias a la cual tiempo es igual a lentitud, corresponde el imperativo difundido, como lugar común, de reducir o anular todas las distancias. El espacio que se interpone entre el principio y el fin de cada recorrido comunicativo se presenta como impedimento. Así como el tiempo intermedio resulta pérdida de tiempo, el espacio intermedio aparece como escombro, desperdicio.

\section{EL ESPACIO URBANO}

Según este modo de sentir el espacio tenemos una muestra en el espacio urbano. Al formar parte del univero comunicativo, el espacio urbano está sometido a su ideo-lógica dominante. El aislamiento del espacio urbano, como, en su interior, el de cada una de sus zonas «periféricas», tiene que ser reducido y, al límite, eliminado, haciendo convertirse al espacio urbano en una parte del conjunto de la red comunicativa de producción, intercambio y consumo.

La reducción de las distancias desde los puntos nodales de esta red (lugar de trabajo, centros comerciales, tiendas y supermercados, oficinas de administrativos, lugares de diversión y de tiempo libre), el estar «bien comunicadas», es criterio de valor (valor de intercambio), sea del espacio urbano total, sea de sus partes individuales. Tal «comunicabilidad», teniendo que darse en cada espacio urbano y en cada una de sus partes, no puede darse, evidentemente, por una vecindad física, «natural», sino por una vecindad tecnológicamente conseguida, «servida» (por redes viales, ferroviarias, metropolitanas, por cadenas televisivas, por líneas telefónicas, telemáticas, por correo electrónico, etcétera). El espacio urbano, por lo tanto, tiene que poder estar completamente abierto a la comunicación; no tiene que contener residuos inaccesibles; tiene que ser completamente transitable, recorrible en cada uno de sus puntos.

El objetivo de la accesibilidad lo más rápida posible a cada punto del espacio urbano y a cada lugar habitado, conlleva que el espacio, como lo que se interpone, como distancia, separación, tenga que ser eliminado. Eso significa expropiación del espacio urbano, transformado en espacio común funcional para el ciclo comunicativo-productivo. Un espacio amorfo, anónimo, indistinto, homologado. La identidad, la diferencia de este espacio se sacrifica a la indiferencia, al rodillo funcional o útil para el intercambio comunicativo. Este sacrificio de la diferencia se hace de acuerdo con la carac- 
terística de lo social en la forma capitalista: lo social como resultado pasivo de intereses separados, como resultado del interés común del intercambio entre sujetos recíprocamente indiferentes, reunidos sólo por una relación entre cosas, entre objetos equiparados, equivalentes, que como tales pueden hacer de mercancía.

El espacio urbano, igual que el espacio que lo une al resto del universo comunicativo, es la negación obligada de la diferencia, de la identidad. Tal negación es la consecuencia de la recíproca indiferencia de las diferencias, de la separación de las identidades individuales o colectivas pasivamente unidas por el interés, padecido o entendido como si fuese común, por el interés de una comunicación que - en cuanto es homologación, uniformización, anulación de diferencias, en cuanto es comunicación que se reproduce a sí misma, comunicación por la comunicación — no comunica nada.

De ello se deriva un sentido de aburrimiento, que ningún interés, diversión, recreo o ebriedad ofrecida en el vacío de la comunicación (vaciado del espacio y del tiempo por su aceleración) podrá borrar. Un sentido de aburrimiento que está en la base de la relación entre el actual espacio urbano, con sus barrios dormitorios, integrados perfectamente sin embargo, en el circuito comunicativo, y la drogadicción juvenil (el enlace entre la droga de la comunicación permitida y la comunicación prohibida de la droga).

El sentido de frustración de la identidad y de la diferencia también es consecuencia de ello. La identidad y la diferencia se obstinan en afirmarse e imponerse todavía más en la misma separación negada por el espacio urbano y por el cambio comunicativo, $\mathrm{y}$, al hacerlo, transforman la actual recíproca indiferencia-entre-diferencias en hostilidad y conflicto con el extraño y con el diferente.

Las diferencias pueden buscarse sólo en el pasado; el presente las anula. Lo que puede aunar y diferenciar y, por lo tanto, individualizar, es un pasado común: la religión, la lengua, la posición territorial, los orígenes, la ascendencia, las raíces, la sangre, el color de la piel. De tal modo, es posible mantener a distancia, con diversos grados de abyección, desde el odio hasta la llamada tolerancia, que el propio territorio, nacional, urbano, de barrio, de cercanía, de relaciones de trabajo y cotidianidad, ha hecho permeable. El único asidero de identidad entre los distintos centros habitados, dentro de un mismo espacio urbano, viene dado por las diferencias religiosas, étnicas, lingüísticas, de pasado cultural, etc. 


\section{EL ESPACIO ENTRE INDIFERENCIA Y DIFERENCIA MUSEI- FICADA}

A la indiferencia del espacio-tiempo presente se contrapone la diferencia del espacio-tiempo pasado. Pero, en conformidad con nuestra forma social basada en la alternativa entre diferencia y negación de la diferencia, la reivindicación de tales diferencias acaba, de hecho, haciendo las veces de polarización de intereses contrapuestos, de justificación de las barreras, de los ghettos, de las exclusiones, expulsiones, y, tal como los acontecimientos actuales testimonian, de linchamientos, de matanzas y genocidios.

Esta identidad por un pasado común es tanto más fuerte como capacidad de autodefensa y tanto más amplia espacialmente, cuando, al identificarse con la nación, con el Estado o con confederaciones de naciones y Estados como la Unión Europea, se contrapone a lo que la actual comunicación mundializada pone en circulación, a pesar de las diferencias y de los confines. La identidad, gracias a un pasado común, puede así mantenerse fuera del espacio que la identifica al migrante, al extracomunitario, a quien está obligado por los desastres provocados por el desarrollo a solicitar acogida en los países desarrollados.

Con respecto a la libre circulación y a la velocidad posible tecnológicamente en los vehículos privados, el mismo espacio urbano aparece como un inconveniente, como una molestia: limitaciones de velocidad, zonas peatonales, circulación ralentizada. El cierre del casco antiguo a la circulación —si los intereses privados por la libre viabilidad de todo el espacio urbano no son tan fuertes como para impedirla- agudiza el anacronismo y la museificación de esta parte con respeto a la exigencia de la comunicación de total apertura, exposición, respecto al espacio. Pero, por otro lado, frente a la indiferenciación, homologación y opacidad del espacio-tiempo, adecuado todo ello a las exigencias de la comunicación actual, como ya hemos observado, la identidad busca la misma posibilidad de reafirmación en lo que, como resto histórico, o como pretendida caracterización «natural», puede constituir diferencia: la tradición, la costumbre, los monumentos y los testimonios del pasado cultural, la lengua y el dialecto, la religión, la etnia. De este modo, las iglesias, los museos, las ruinas, el casco antiguo se conservan como elementos de caracterización, de individualización de un espacio urbano (hasta el punto de ser el elemento de atracción turística valorizable en el circuito comunicativo), que, por otra parte, se ha hecho anónimo e indistinto con respecto a los demás espacios urbanos. 


\section{VIABILIDAD DEL ESPACIO Y DISPONIBILIDAD DE MEDIOS PERSONALES DE CIRCULACIÓN}

La separación negada por la intromisión en la vida privada de los medios y canales de comunicación y por los programas de circulación y consumo se compensa por la ilusoria oferta tecnológica de progresivas mayores libertades individuales de comunicación, asimismo disponibles para todos, sea dentro del espacio urbano, sea en el ámbito de la entera red comunicativa. Es la ilusión del libre mercado, del trabajo libre, de la libre elección de trabajo, del intercambio equitativo entre sueldo y fuerza de trabajo, ilusión que se refleja, en el interés de la reproducción ampliada del ciclo productivo-comunicativo, en la aparición en el mercado de medios de comunicación disponibles por cada uno, individual y libremente.

La libertad individual de comunicación se transforma de nuevo en la accesibilidad más rápida que sea posible del individuo (por ejemplo, gracias al teléfono portátil), y, por lo tanto, en una ulterior intromisión de la red comunicativa en el ámbito de la «vida privada». La libertad de comunicación es efectivamente constricción a formar parte de esa red comunicativa que le permite persistir y extenderse al ciclo productivo — con la presión para respetar sus ritmos, para adaptarse a su velocidad-.

La idea de la separación del propio cuerpo, del cierre de la morada propia, de la autonomía del propio espacio circundante, idea que la identidad personal necesita en nuestra forma social para poder circunscribir los «intereses personales» con respecto a los «intereses ajenos», sirve de barrera al mismo tiempo a la conciencia de las consecuencias negativas de la inevitable implicación, del compromiso, de todos en el ciclo comunicativo-productivo. Impide, por ejemplo, una conciencia auténticamente operativa del coste en salud de la libertad de contar individualmente con medios privados de transporte. Coste en salud por la polución, por la invivilidad del espacio urbano, debido a las emisiones automovilísticas y a los accidentes de tráfico. El contraste entre productividad y salud en la fábrica, en su interior y en su espacio circundante se presenta en el espacio urbano. Dicho sea de pasada: el espacio circundante de la fábrica, dada la tendencia a aumentar de su nocividad por el desarrollo tecnológico, es cada vez más extenso.

Pero la libertad de contar con medios de circulación privados, individuales (más bien individualizados: tipo de automóvil, cilindrada, color...), libertad que justifica su producción y que incrementa los riesgos para la salud en el espacio urbano por su polución y por la degradación consiguiente, se 
transforma (se disfraza) paradójicamente en soluciones ofrecidas a los individuos, privadamente. Soluciones para quien quiera optar por el vaivén, por habitar fuera de la ciudad, o para quien quiera huir ocasionalmente (durante el tiempo libre, los fines de semana, durante las vacaciones, etc.) del «ambiente insano» de la ciudad.

Obviamente, habitar fuera de la ciudad sirve también para sustraerse a los costes elevadísimos de los alojamientos en los centros más cotizados (por su valor de intercambio) debido a su inserción en la red comunicativa.

\section{MIGRACIÓN Y ALTERIDAD IRREDUCIBLE A LA COMUNIDAD DEL TRABAJO}

Por lo que atañe a su carácter de incómodo testigo, el fenómeno de la «droga» puede ser parangonado con otro fenómeno llamativo de nuestro tiempo, el de la migración. Ambos «embarazan» al sistema. En efecto, la migración, a diferencia de la tradicional emigración, que puede ser absorbida y convertida en funcional para el sistema, no es controlable más que con la represión y la expulsión.

Con respecto al mundo normal de las mercancías y de la compra-venta del trabajo como mercancía, con respecto a las mercancías «domesticadas», la «droga» y la migración revelan las vergüenzas de la mercantilización generalizada y también su impotencia, además de la brutalidad del sistema capitalista. Es sintomático que, sea con respecto a la «droga», sea con respecto a la migración, el Estado tenga que recurrir a la fuerza, y que se desencadenen en la misma sociedad civil comportamientos de intolerancia: el «drogadicto» y el migrante (el «extracomunitario») se emparejan por la misma actitud con que se les trata, actitud de defensa y, por lo tanto, de exclusión o de eliminación provocada, con la consiguiente denuncia (la denuncia que constituye su misma presencia), con la consiguiente denuncia de las contradicciones insolubles de este sistema.

Las migraciones son el fenómeno de la otra realidad no englobable en la totalidad más amplia de la que Europa forma parte, es decir, de la totalidad del sistema capitalista que, en su difusión ya planetaria, contiene en su interior zonas de alteridad absoluta, lo mismo da que se llamen subdesarrollo, países en vías de desarrollo, países en expansión, tercer mundo o sur del mundo.

Nombrar el otro absoluto es difícil, a veces imposible: el término extracomunitario que la Europa unida ha querido acuñar forma parte de esa serie 
de nombres con los que se intenta nombrar una alteridad que no se deja identificar. Se trata del otro del desarrollo capitalista que no puede transformarse en desarrollo porque el desarrollo necesita constitutivamente de él.

Una alteridad en el corazón mismo de la identidad del capitalismo. Generada por este último, y aún así, irreduciblemente otra. Una necesidad y una amenaza.

Una amenaza que hoy se presenta no tanto como oposición, como lucha al capitalismo, sino bajo forma de petición de acogida. Una petición de masas, en esto se diferencia del fenómeno precedente, detectable en las migraciones, una petición enorme que, como tal, coloca en el banco de los acusados la identidad que, no sólo no puede satisfacerla, sino ni siquiera admitirla. El subdesarrollo englobado en el sistema capitalista mundial solicita hoy acogida en los países desarrollados: éste es el fenómeno de las migraciones.

El fenómeno de la emigración, desde zonas menos desarrolladas a zonas más desarrolladas, es un fenómeno controlable, contenible. Es un fenómeno funcional al desarrollo. Pero la migración va más allá del hecho emigratorio. No nace como traslado de fuerza de trabajo que, aunque antagonista del capital, es su parte complementaria, sino como desplazamiento de personas desde las zonas de subdesarrollo, que solicitan acogida en los países desarrollados, que solicitan un puesto, no sólo un puesto de trabajo. Una solicitud de acogida que es también una pregunta, donde se solicita una justificación por parte de quien ocupa un lugar en el desarrollo, no simplemente un puesto de trabajo, por parte de quien ya tiene una colocación y ha creído hasta ese momento que no tenía que rendir cuentas a nadie.

En la migración no es la alteridad de la fuerza de trabajo, que es una alteridad relativa interna a la comunidad, o si es externa, englobable en ella, la que solicita y pregunta, sino una alteridad absoluta que pone en discusión la identidad con su misma solicitud de acogida. La cual es por sí misma inaceptable, y suena sin querer, como un acto de acusación porque evidencia en la identidad la mala conciencia de la buena conciencia, porque pone de manifiesto en el capitalismo el subdesarrollo, la opresión, la segregación, la pobreza, el hambre, la enfermedad, la muerte, la guerra, como su manifestación irreducible.

La solicitud de acogida de la migración es la solicitud de una alteridad inasimilable por parte de la comunidad, es solicitud absoluta de extracomunitario; es solicitud que no puede ser traducida en solicitud de puesto de trabajo y ser asimilada a la del parado. Es la solicitud de quien no encuentra un 
puesto en la totalidad, en el orden del idéntico: de quien no puede ser asimilado por la dialéctica interna de la identidad del capital, solicitud, que por su absoluta alteridad, no forma parte del plan preestablecido de una dialéctica monológica, sino que expone al riesgo de la dialéctica dialógica.

Tal solicitud no se pronuncia ni siquiera en nombre de los «derechos humanos» que existen históricamente, y por lo tanto «naturalmente», cuando se entienden de forma jurídica y naturalista, los derechos de la identidad. Aquí se trata, en cambio, de los derechos de un humanismo del otro, de otro hombre, que no es de este mundo, se trata de los derechos de la alteridad. Derechos de la diferencia. Pero de una diferencia que no tiene género, que no es relativa, que no es interior a la identidad comunitaria y a su dialéctica. Una diferencia, en cambio, de extracomunitario.

\section{SENTIRSE EXTRACOMUNITARIO}

Hoy la comunidad, que el capitalismo con su lógica de la identidad ha producido, se pone en discusión, no tanto por el conflicto de intereses diferentes de las identidades de las que es el resultado. Por cuánto exacerbados y violentos puedan mostrarse dichos conflictos forman parte de la misma lógica del capitalismo, y son, de algún modo, de tipo fisiológico y también funcional al mismo, que se pueden resolver por ejemplo, con la extrema ratio de la guerra, producto y mercancía, ella misma, del sistema capitalista.

La comunidad que el capitalismo puede producir, dada su lógica de la identidad, se encuentra puesta en discusión por la alteridad extracomunitaria, que con la solicitud de acogida de las migraciones reduce cada vez más la posibilidad de dar disculpas, la posibilidad de la buena conciencia, la posibilidad de la indiferencia, sobre la que la identidad ha construido la diferencia.

Por una parte, la reivindicación de la diferencia de género (sexo, clase, región, etnia, religión, etc..), la reivindicación de los derechos de la identidad, o bien de su alteridad correspondiente, que es reivindicación de individualidades diferentes en base al género; por otra, la solicitud de acogida como reivindicación de los derechos de la alteridad, una alteridad fuera de cualquier género, a pesar de los esfuerzos que podamos hacer para englobarla en el género, de tal forma que el rechazo, la marginación, la expulsión puedan encontrar una justificación; una alteridad absoluta, de personas aisladas y no de individuos correspondientes a un género. 
El extracomunitario exige una respuesta, lo que comporta poner en discusión la identidad comunitaria y sus leyes, poner en discusión la lógica de la identidad.

Una respuesta que puede venir solamente de nuestra misma alteridad, de nuestro mismo ser otros respecto a esa lógica, y respecto a nuestra comunidad - la comunidad de Raza, Historia, Etnia, Nación, Región, Religión, Partido, Individuo-, la comunidad de la diferencia-indiferencia, de las disculpas, de la responsabilidad delimitada, de la negación de la no in-diferencia.

La solicitud de acogida del extracomunitario puede recibir respuesta sólo de nuestra misma situación de extracomunitarios respecto a nosotros mismos (Kristeva), respecto a nuestras comunidades de pertenencia, sean grandes o pequeñas, colectivas o individuales, en las que nuestra misma alteridad de individuos únicos se encuentra segregada, excluida, pero no destruida por completo. La petición del extracomunitario solicita que la comunidad se abra hacia la alteridad, solicita una acogida de la alteridad. Una amenaza para la identidad y para las semejanzas comunitarias construidas sobre ella, una amenaza para la reproducción de la forma social capitalista. ¿Cuántas medidas — políticas, económicas, jurídicas- harán falta para denegarla? ¿Cuántos ejércitos, cuántas justificaciones, cuántas disculpas? Pero, al mismo tiempo es una ocasión - ¿la última? - de liberación para nuestra alteridad, una ocasión para existir como individuos, sin el caparazón de la identidad individual, sin la identificación en base al género, sin la intercambiabilidad que dicha identificación conlleva; una ocasión para existir como individuos, convirtiéndonos en únicos por la no indiferencia, por la aislada responsabilidad sin disculpas hacia el otro. Una oportunidad para poder superar lo social como lugar de la recíproca indiferencia y lugar de encuentro y conflicto de intereses privados. Una oportunidad para abrir todo espacio comunitario al extracomunitario. Comunidades estructuralmente extracomunitarias, orientadas hacia su continua destotalización. Lejos de la categoría de la identidad. Para que se vuelva a fundar sobre la alteridad.

\section{ALTERIDAD Y PROXIMIDAD ENTRE MIGRACIÓN Y PARO}

Ambos excedentes, el parado y el migrante, constituyen un límite no eliminable de la posibilidad de explotación del trabajo libre. Esta condición los hace pares. A este acercamiento de hecho se contrapone una diferencia de derecho, la diferencia de la pertenencia del primero, y de la no pertenencia 
del segundo, al territorio nacional, la diferencia entre «comunitario» $\mathrm{y}$ «extracomunitario».

Hay, sin embargo, un objetivo proceso de acercamiento entre migrante y parado, basado no ya en el viejo internacionalismo que dependía de la abstracción (concreta) del trabajo. El carácter ilusorio de tal internacionalismo se ha evidenciado muy bien cada vez que se ha tenido que confrontar con la solución realista de la guerra: necesariamente, porque el trabajo en general, el trabajo abstracto, forma parte de la misma realidad en la que la guerra logra resultar siempre la solución más realista, aunque sea como extrema ratio.

Pero estamos hablando del objetivo proceso de acercamiento entre migrante (extracomunitario) y parado (comunitario), debido precisamente, por el contrario, a su común irreductibilidad a tal (concreta) abstracción, que permite un encuentro fuera de la identidad, un encuentro de alteridad.

Si es verdad que el paro actual no es algo momentáneo, coyuntural, sino un hecho estructural que representa un cambio decisivo en las relaciones sociales de producción, porque inicia el fin del «trabajo libre», del intercambio trabajosalario, se está creando una conexión muy estrecha entre paro y migración. En ambos casos hay una expulsión que es estructural en el sistema. Ni el paro ni la migración, fenómenos ambos muy difusos en nuestro tiempo, pueden ser absorbidos, convertidos en funcionales para el sistema de producción que los ha engendrado. Ambas excedencias, el parado y el migrante, constituyen un límite no eliminable de la posibilidad de explotación del trabajo libre.

A este acercamiento contrapone actualmente el parado «comunitario» las tentativas, en gran parte ilusorias, de recuperación, en formas diferentes, de la identidad amenazada, y la defensa del derecho al propio trabajo, con los consiguientes reflujos de racismo, y con la expulsión, si no ya con la pura eliminación física, del extracomunitario. Pero, guste o no guste, la realidad es que el sistema capitalista en su estado actual de producción transforma al mismo comunitario parado, como excedencia, en migrante, es decir, en individuo constitutivamente inútil para el proceso productivo, un individuo que, a diferencia del emigrante y del parado tradicional, no puede ser absorbido.

Reconocer esto significa reorganizar la cuestión de la defensa del trabajo, que o divide apelando a la diferencia, y engendra actitudes racistas y étniconacionalistas, o unifica, según un internacionalismo basado sobre la indiferencia del trabajo abstracto - internacionalismo cada vez más de capa caída por la crisis misma del trabajo abstracto, que tampoco permite ya la identidad genérica, y enajenada, de clase obrera-. 
El reconocimiento del acercamiento del paro a la migración, del parado al extracomunitario, supone, en cambio, la reorganización de lo social, convirtiendo la liberación del trabajo abstracto, del trabajo indiferenciado, en tiempo disponible para la alteridad, y convirtiendo el acrecentamiento de la producción - hecho posible por el desarrollo de las fuerzas productivas- en trabajo útil para las necesidades de todos.

Esta perspectiva es la de una forma social postcapitalista, global como el sistema capitalista del que deriva, pero, a diferencia de éste, capaz de abrir lo social a la alteridad, de librarse de la obsesión por la identidad, de realizarse, por lo tanto, como no identitaria, como extracomunitaria a sí misma, como algo distinto, sin confines, territorios, pertenencias, raíces, no ya basadas en el trabajo, útil o abstracto, sino en el tiempo disponible para la alteridad; no en la diferencia ni en la indiferencia, sino en en la diferencia no-indiferente, que hace de cada ser humano de la tierra un prójimo para cada uno de los demás seres humanos.

\section{LA POSIBILIDAD DE SENTIRSE EN EL MUNDO PERO NO DEL MUNDO DE LA GLOBALIZACIÓN}

El Mundo de la mundialización, de la comunicación-producción mundializada está indisolublemente ligado a la política, ya en cuanto proyección, en cuanto plano, en cuanto espacio de satisfacción de necesidades; a la política como visión totalizadora y organización funcional, como estrategia de la productividad, de la eficiencia, como actitud adherente a la realidad, como garantía en el conatus essendi, como mediación del inter-esse tanto de sujetos individuales como de sujetos colectivos, como conciencia y gestión del futuro a partir de la visión realista del presente y a través de la readaptación a ella del pasado, como administración racional de la duración, como economía del persistir, del progresar en el ser, a cualquier coste. También al precio de la guerra. La guerra forma parte del Mundo, está prevista en él, forma parte de su lógica, de la ontología del conatus essendi. El Mundo prevé la guerra, porque, al estar basado constitutivamente en la identidad, utiliza funcionalmente - para el mantenimiento, para la consolidación, para la duración, para la reproducción, ampliada, de lo mismo - todo lo que es otredad, todo lo que es el otro; está listo, está predispuesto al sacrificio de la alteridad al servicio de la identidad.

La paz es momentáneo descanso, repliegue de fuerzas, la tregua que sigue a la guerra, la necesaria «vuelta a casa», la preparación también para una amenaza eficaz de volver a recurrir a ella, del mismo modo en que el des- 
canso, el tiempo libre, la noche, están al servicio de la reanudación del trabajo, de las necesidades del día. El trabajo y la guerra: la guerra como evidente «trabajo colectivo» en las formas de producción precapitalista. La paz vive por y para la guerra, así como el descanso, la noche, viven por y para el trabajo, para el día.

La cuestión - cuestión filosófica, la única a partir de la que es posible la crítica efectiva de la comunicación mundializada - es, si acaso, que no hay otro sentido que en el Mundo y para el Mundo, si acaso lo específicamente humano no emana del espacio y del tiempo de la ontología, si acaso no existen relaciones irreductibles a la categoría de la identidad y que no tengan nada que ver con las relaciones entre sujeto y objeto, o de intercambio, de equiparación, de función, de interés, de productividad; si acaso no hay relaciones inter-humanas que sean todo lo contrario, otras relaciones inter-humanas, pero que no pertenezcan a otro mundo o a otra dimensión del ser, ni sean sólo alternativas, en tanto modalidades de ser de otra manera.

Esas relaciones formarían parte, muy al contrario, de lo que Lévinas llama lo distinto del ser, lo que está fuera de la ontología, fuera del Mundo. Son, sin embargo, relaciones materiales y terrenales, a las que está abierto el propio cuerpo: una transcendencia terrenal con respecto al Mundo, un sentido distinto con respecto a lo intra-mundano, un humanismo diferente del de la identidad, diferente del humanismo del ser del hombre; al contrario, un humanismo de la alteridad, de lo distinto de ser.

Estas relaciones son dialógicas, pero no en el sentido de diálogo formal o de diálogo como consenso, como convenir, como acuerdo, o como dialéctica en la que las contradicciones se superan en la síntesis. Diálogo, en cambio, como exposición - peligrosa - a la alteridad, como hibridación de la identidad, como ruptura del monologismo de la globalización. Una crítica de la comunicación mundializada se presenta, en consecuencia, como una crítica de la razón dialógica.

(Traducción de José María Nadal)

\section{REFERENCIAS BIBLIOGRÁFICAS}

Athanor. Semiotica, filosofia, arte, letteratura. Mondo 6, 1995.

Athanor. Semiotica, filosofia, arte, letteratura. Vita, Augusto Ponzio (ed.), XIII, 5, 2002. 
Athanor. Semiotica, filosofia, arte, letteratura. Nero, Susan Petrilli (ed.), XIV, 6, 2003.

Athanor. Semiotica, filosofia, arte, letteratura. Lavoro immateriale, Susan Petrilli (ed.), XIV, 7, 2003-2004.

Athanor. Semiotica, filosofia, arte, letteratura. Il dono/The Gift. A Feminist Analysis, Genevieve Vaughan (ed.), XV, 8, 2004.

Delors, J. (1994). Libro bianco. Crescita, competività, occupazione (White Book. Growth, competitivity, employment). Milán: Il Saggiatore.

Eco, U.; Gandelman, C.; Kristeva, J. y otros (1993). Migrazioni. Athanor. Semiotica, filosofia, arte, letteratura 4.

European Commission (véase también Delors, J.). Documents and Communications of the European Commission: www.europa.eu.int.

Foucault, M. (1977). Power/Knowledge. Hemel Hempstead: Harvester.

- (1988). Technologies of the Self. A Seminar. Amherst: The University of Massachusetts Press.

Gorz, A. (1988). Métamorphoses du travail. París: Galilée.

Gramsci, A. (1975). Quaderni dal carcere. Turín: Einaudi.

Kant, I. (1998 [1781-87]). Critique of Pure Reason, Paul Guyer y Allen W. Wood (ed. y trad.). Cambridge: Cambridge University Press.

LÉvinas, E. (1961). Totalité et infini. La Haya: Nijhoff.

- (1972). Humanisme de l'autre homme. Montpellier: Fata Morgana.

- (1974). Autrement qu'être ou au-dela de l'essence. La Haya: Nijhoff.

- (1987a). Hors Sujet. Montepellier: Fata Morgana.

- (1987b). Collected Philosophical Papers, A. Lingis (trad. y ed.). Dordrecht: Martinus Nijhoff Publishers.

MARX, K. (1953 [1857-58]). Grundrisse der Kritik der politischen Ökonomie. Berlín: Dietz-Verlag.

Marx, K. y Engels, F. (1968). Selected Works in One Volume. London: Lawrence and Wishart.

PAsolini, P. P. (1990). Scritti corsari. Milán: Garzanti. 
PetriLli, S. (1998). Teoria dei segni e del linguaggio. Bari: Graphis (Nueva ed. 2001).

- (ed.) (1999). Semiotic Studies in Bari. S-Europen Journal for Semiotic Studies. Special Issue, vol. II-4.

- (2003a). «Sebeok's Semiosic Universe and Global Semiotics.»Cybernetics \& Human Knowing. A Journal of Second-order Cybernetics, Autopoiesis and Cyber-Semiotics. Thomas Sebeok and the Biosemiotic Legacy, S. Brier (ed.), vol. 10, 1, 61-79.

- (2003b). «The Sign-Machine: Linguistic Work and World Communication.» Quaderni del Dipartimento di Pratiche Linguistiche e Analisi di Testi, PLAT 2, 347-366, Università degli Studi di Bari. Bari: Edizioni dal Sud.

- (2004a). «Semioethics, Subjectivity, and Communication: For the Humanism of Otherness.»Ideology, logic, and dialogue in semioethic perspective. Semiotica. Journal of the International Association for Semiotic Studies 148-1/4, Special Issue, Susan Petrilli (ed.), 69-92.

- (2004b). «Human Responsibility in the Universe of 'Global Semiotics'.» Semiotica. Journal of the International Association for Semiotic Studies 150-1/4, 23-38. Special Issue, Eero Tarasti (ed.).

- (2004c). «Gift-giving, mother-sense and subjectivity in Victoria Welby. A study in semioethics.»Il dono/The Gift. A Feminist Analysis. Athanor. Semiotica, Filosofia, Arte, Letteratura XV, n. s. n. 8, 179-198, Genevieve Vaughan (ed.).

- (2004d). «The Responsibility of Power and the Power of Responsibility: From the 'Semiotic' to the 'Semioethic' Animal.» En Gloria Withalm \& Josef Wallmannsberger (Hg./Eds.), Macht der Zeichen, Zeichen der Macht. / Signs of Power, Power of Signs. Essays in Honor of Jeff Bernard, 103-119, INST (www.inst.at). Wien.

- (2005). Percorsi della semiotica. Bari: Graphis.

Petrilli, S. y Ponzio, A. (1998). Signs of Research on Signs. Semiotische Berichte. Österreichschen Gesellschaft für Semiotik, Special Issue Jg. $22,3 / 4$.

- (2000). Il sentire nella comunicazione globale. Roma: Meltemi.

- (2001). Sebeok and the Signs of Life. London: Icon Books. 
— (2002). «Sign Vehicles for Semiotic Travels: Two New Handbooks.»Semiotica. Journal of the International Association for Semiotic Studies 141-1/4, 203-350.

- (2003a). Semioetica. Roma: Meltemi.

- (ed.) (2003b). Linguaggi. Bari: Laterza.

- (2004). «Global Communication, Proximity and Responsibility: Beyond the Logic of Identity.» Semiotica. Journal of the International Association for Semiotic Studies 150-1/4, 151-167. Special Issue, Eero Tarasti (ed.).

- (2005). Semiotics Unbounded. Interpretive Routes in the Open Network of Signs. Toronto: Toronto University Press.

Ponzio, A. (1990). Man as a Sign. Essays on the Philosophy of Language, S. Petrilli (ed.). Berlín: Mouton de Gruyter.

- (1992). Production linguistique et idéologie sociale. Candiac (Québec): Les Éditions Balzac.

— (1993). Signs, Dialogue, and Ideology, S. Petrilli (ed.). Ámsterdam: John Benjamins.

- (1995). La differenza non indifferente. Comunicazione, migrazione, guerra. Milán: Mimesis (2. ${ }^{a}$ ed. 2002).

- (1996). Sujet et altérité. Sur Emmanuel Levinas. París: L'Harmattan.

- (1997). Elogio dell' infunzionale. Critica dell' ideologia della produttività. Roma: Castelvecchi.

- (1999). La comunicazione. Bari: Graphis.

- (2003a). I segni tra globalità e infinità. Per la critica della comunicazione globale. Bari: Cacucci.

- (2003b). Tra semiotica e letteratura. Introduzione a Michail Bachtin. Milán: Bompiani.

- (2004a). Semiotica e dialettica. Bari: Edizioni dal Sud.

- (2004b). Linguistica generale, scrittura letteraria e traduzione. Florencia: Guerra.

Ponzio, A.; Calefato, P. y Petrilli, S. (1994). Fondamenti di filosofia del linguaggio. Roma y Bari: Laterza (Nueva ed. ${ }^{\circ} 1999$ ). 
Ponzio, A.; Petrilli, S. y Ponzio, J. (2005). Reasoning with Lévinas. Ottawa: Legas.

RIFKIN, J. (1995). The End of Work. London: Penguin (2. ${ }^{\mathrm{a}}$ ed. $\left.{ }^{\circ} 2000\right)$.

- (1998). The Biotech Century. New York: Penguin-Putman.

Rimbaud, A. (1995). Una stagione all' inferno, G.-A. Bertozzi (ed.). Roma: Newton Compton.

Rossi-Landi, F. (1977). Linguistics and Economics. La Haya: Mouton.

- (1982). Ideologia. Roma: Meltemi (Nueva ed. ${ }^{\circ}$ 2005).

- (1985). Metodica filosofica e scienza dei segni. Milán: Bompiani.

— (1992). Between Signs and Non-Signs, S. Petrilli (ed.). Ámsterdam: John Benjamins.

Sebeor, T. A. (1994b). Signs. An Introduction to Semiotics. Toronto: Toronto University Press (Nueva ed. ${ }^{\circ}$ 2001).

— (2001). Global Semiotics. Bloomington: Indiana University Press.

Sebeor, T. A.; Petrilli, S. y Ponzio, A. (2001). Semiotica dell'io. Roma: Meltemi.

ZANOTELl, A. (1996). Leggere l'impero. Il potere tra l'Apocalisse e l'Esodo. Molfetta (Bari): Edizioni La Meridiana. 